\title{
Fibules digitées de tradition " danubienne " de l'époque des Grandes Migrations, découvertes en Gaule du Sud
}

\author{
Michel KAZANSKI*
}

En Gaule du Sud durant la deuxième moitié du V ${ }^{e}$ siècle et au $\mathrm{VI}^{\mathrm{e}}$ siècle se diffusent les fibules digitées dont certaines appartiennent à la tradition vestimentaire « danubienne» des Germains orientaux et caractérisent « la classe moyenne » des sociétés barbares du début du Moyen Âge. Les fibules de tradition danubienne représentent le témoignage archéologique des contacts entre la Gaule du Sud et les royaumes germaniques orientaux du Danube moyen et d'Italie. Ces contacts, à part les relations avec l'Italie ostrogothique, sont peu attestés par les auteurs anciens, car le matériel archéologique et les sources écrites montrent l'histoire d'une façon différente.

Mots-clés : fibules, époque des Grandes Migrations, Gaule du Sud, Germains orientaux, Wisigoths, Danube.

\section{INTRODUCTION}

En Gaule du Sud durant la deuxième moitié du $\mathrm{V}^{\mathrm{e}}$ siècle et au $\mathrm{VI}^{\mathrm{e}}$ siècle, comme partout en Europe, se diffusent les fibules digitées. On peut y distinguer plusieurs types « septentrionaux », répandus en Gaule du Nord (voir par ex. Koch 1998, Karten 4,5,8,9,10 etc. ; Stutz 2000, fig. 3.6-9, 4.1,2). D'autres fibules, à pied losangique et à tête semi-circulaire, appartiennent à la tradition germanique orientale (fig. 1). Les fibules de ce type, ornées d'un décor végétal ou géométrique en relief, apparaissent dans la région du Danube moyen au deuxième tiers du Ve siècle (Tejral 2002, 321). Parmi les types danubiens les plus anciens il faut citer celui de Sokolnice, portant un décor en volutes sur le pied et sur la tête (fig. 2.4). Ces agrafes apparaissent dans la région du Danube moyen au milieu du Ve siècle et donnent des ramifications nombreuses dans la deuxième moitié du Ve -début du VIe siècle (par ex. fig. 2.5,10,11) (Tejral 1997, 349, 359, Abb. 28. 13,14; Tejral 1997a, 144, fig . 10.7,8; Tejral 2002, 321, Abb. 6.7,8 ; Tejral 2008, 258, Abb. 5.16 ; Menke 1986, 247). A la même époque se diffusent les fibules danubiennes portant un décor radial sur la tête et en forme de losanges sur le pied (Tejral 2002, 321, Abb. 6.1,2,5,9; Tejral 2008, 258, Abb. 5.1-3) (fig. 2.1-3).

Les fibules digitées à tête semi-circulaire et pied losangique font partie du costume féminin en Italie ostrogothique, ainsi que dans les royaumes germaniques du Danube moyen (Gépides, Suèves, Skires, Ruges etc.), chez les Goths de Crimée ainsi qu'en Espagne wisigothique. Elles étaient portées sur les épaules, souvent accompagnées d'une grande plaque-boucle de ceinture (Bierbrauer 1975, 71-83). Il s'agit assurément d'un costume féminin « ethnographique » de tradition germanique orientale - un des rares traits dans les royaumes romano-germaniques d'Occident permettant d'identifier la population qui se réclamait de l'identité «gothique». Notre propos consiste à présenter les fibules de ce groupe (1), découvertes en Gaule du Sud (2), dans la zone des royaumes wisigothique et ostrogothique. L'étude de leur origine précise contribuera à nos connaissances des contacts entre les royaumes gothiques d'Occident et l'Europe centrale. Il faut souligner la rareté de ces fibules même à l'intérieur des sites funéraires fouillés, ce qui montre que dans les anciennes communautés méditerranéennes, les porteurs, ou plutôt les porteuses de ce costume étaient très minoritaires. D'autre part, ces personnes n'étaient pas toutes obligatoirement d'origine gothique. En effet, dans les royaumes barbares d'Occident le costume féminin germanique, en quelque sorte « ethnographique » à l'origine, devient progressivement un signe distinctif de position sociale (voir en particulier von Rummel 2007, 401-406).

\footnotetext{
* Directeur de Recherche (DR 2) au CNRS - UMR 8167 « Orient et Méditerranée », Collège de France, 52, rue du Cardinal Lemoine, Paris-75005 ; michel.kazanski@college-de-france.fr.

Je remercie Patrick Périn pour la lecture critique et amicale de cette étude. Remerciements à Guy Barruol et Michel Feugère pour la critique constructive. ${ }^{2}$ Certaines fibules digitées du Midi, comme l'exemplaire de Figaret à Guzargues (Hérault) ou encore de Routier (Aude) (Landes 1988, N 9,11) n'apparaissent pas dans cette étude parce que je ne connais pas leurs parallèles proches dans la région danubienne.
} 


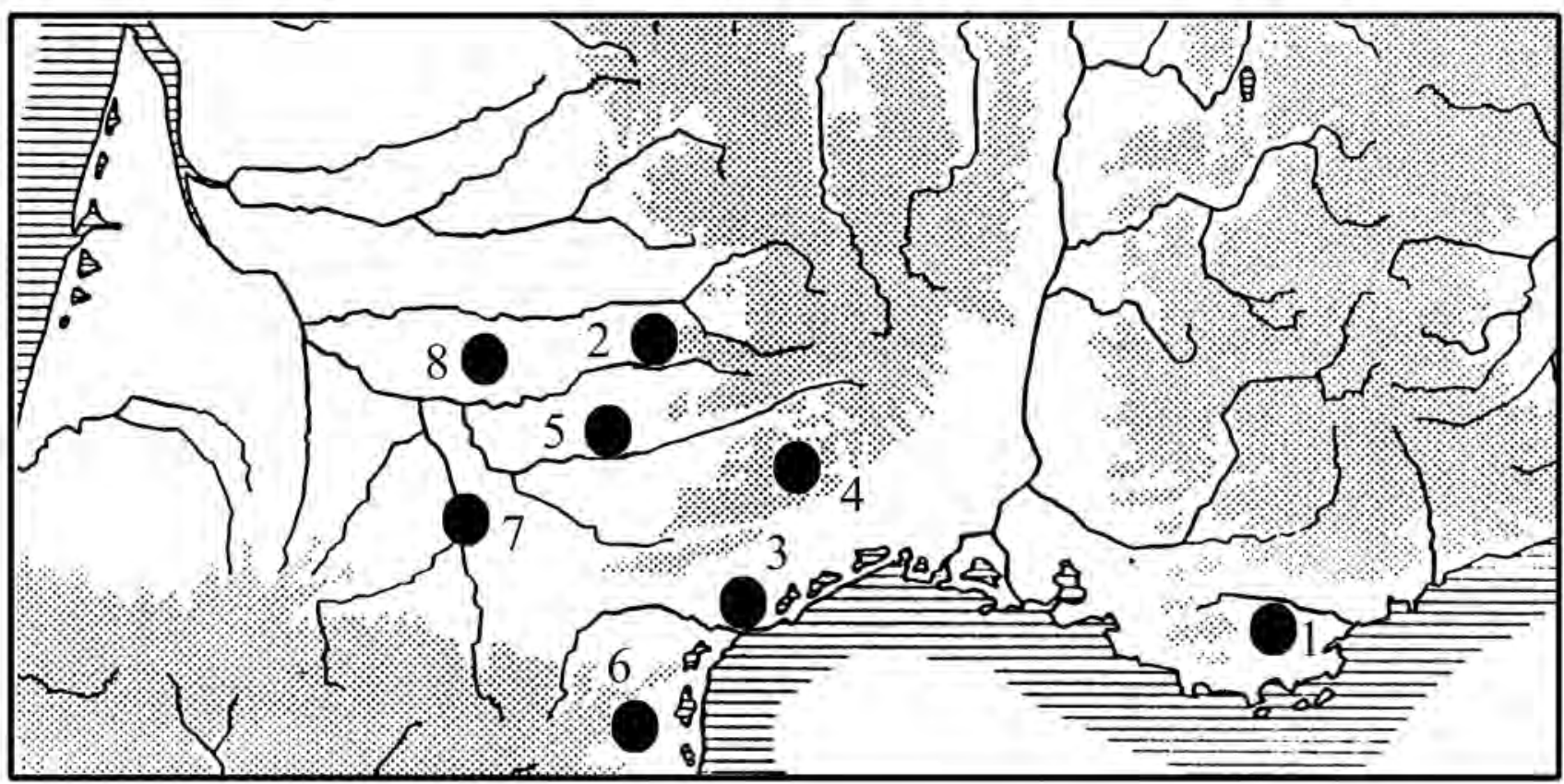

Fig. 1 :.Les découvertes des fibules digitées de tradition germanique orientale en Gaule du Sud. 1 : Taradeau-Saint-Martin; 2 : Souyri; 3: Sérignan; 4: Saint-Affrique; 5: Laroque-Castayrols; 6 : Estagel ; 7 : Toulouse ; 8 : Montheils.

\section{LES PETITES FIBULES DU TYPE DE FICAROLO (fig. 3).}

\section{Taradieu-Saint-Martin (Var).}

Ce type, daté de la deuxième moitié du $V^{e}$ et du début $\mathrm{du} \mathrm{VI}^{\mathrm{e}}$ siècle a été étudié grâce aux découvertes effectuées en Italie ostrogothique, dans la tombe 4 de Ficarolo (Bierbrauer et al. 1993, 322-324) (fig. 3.4). Ce sont les petites agrafes (moins de $10 \mathrm{~cm}$ de la longueur), à trois doigts, qui présentent un décor à quatre volutes dans la partie centrale du pied et à deux volutes opposées sur la tête, parfois séparées par un triangle en taille biseautée ou par une nervure verticale. L'anse est divisée en deux champs par une ligne longitudinale en relief. Le pied se termine par un masque zoomorphe. Enfin, les bordures du pied et de la tête, ainsi que de l'anse, portent parfois un décor en petits triangles poinçonnés.

Une pièce dérivée de ce type provient de TaradieuSaint-Martin, en Provence (Var, commune de Taradeau), c'est-à-dire du territoire du royaume italo-osthrogothique (fig. 1.1). Lors de travaux effectués dans la chapelle de Saint-Martin (construite au XII ${ }^{\mathrm{e}}$ siècle), on a mis au jour deux niveaux d'inhumations - gallo-romaine en coffrage en tuile et celui du Haut Moyen Âge. Les inhumations médiévales étaient déposées selon l'axe Nord-Sud, en terre libre. La fibule en question est le seul objet conservé de cette découverte. La fibule est faite de bronze étamé, sa longueur est de 6,2 cm (Boyer 1971, 153, 154, fig. 6.3 ; première notice dans Gallia 27, 1969 , 145). Seulement deux des cinq doigts sont conservés, le décor végétal du pied et de la tête est dégradé, l'extrémité du pied est ornée d'un masque zoomorphe (fig. 3.1).
Les petites fibules de ce type se diffusent dans une zone assez large. En premier lieu il faut évoquer des pièces découvertes en Gaule de l'Est, provenant de Brochon (fig. 3.2), de Chaussin (fig. 3.3) (Vallet, Kazanski, De Pirey 1995, 112, 117, fig. 4.5 ; Koch 1998, 222-224, No 202, 238, Taf. 34.1,2), de BâleGotterbarmweg, tombe 22 (fig. 3.6) (Vogt 1930, Taf. 10.XXII.3,4), de Bâle -Kleinhüningen, tombe 75 (fig. 3.5) (Giesler-Müller 1992, Taf. 67.3,4), où elles font partie du groupe B des fibules, d'origine germanique orientale, selon M. Martin (Martin 2002, 198, 199, Abb. 3). D'autre part, ces fibules ont été mises au jour à Ficarolo, tombe 4 (fig. 3.4), déjà citée, en Italie ostrogothique (Bierbrauer et al. 1993, 322-324 ; I Goti 1994, Cat. $n^{\circ}$. III.21, fig. III.61) et dans la région du Danube moyen, quelque part en «Hongrie » (Csallány 1961, Taf. CCIII.7), ainsi que dans la nécropole de CsongrádKettoshalöm (fig. 3.8), sur le territoire des Gépides en Hongrie orientale (Csallány 1961, Taf. CCXI.14,15 ; Germanen 1987, 233, V.28), à Bojná (fig. 3.10), dans un habitat fortifié sur le territoire de la Slovaquie (Pieta 2006, obr. 3.1), à Stehlčeves (une paire), dans une tombe (fig. 3.11), sur le territoire de la Bohême (Svoboda 1975, obr. 10) ou encore de Stössen, tombe 69 (fig. 3.9), en Thuringe (Bierbrauer 1975, Taf. 70.5). Ces fibules, datées de la deuxième moitié du $\mathrm{V}^{\mathrm{e}}$ et du début du $\mathrm{VI}^{\mathrm{e}}$ siècle (Bierbrauer et al. 1993, 322-324) sont les plus proches de celle de Taradeau-Saint-Martin. Les fibules digitées à pied losangique et tête semi-circulaire portant un décor végétal en volutes, le type Ficarolo y compris, remontent au type danubien dit de Sokolnice (voir supra) (3). Les pièces provenant de la tombe isolée à 
Dabronc-Otvöspuszta (fig. 3.7), en Hongrie occidentale, représentent peut-être la version intermédiaire entre les types Sokolnice et Ficarolo. En tout cas ces agrafes, bien travaillées, sont le prototype direct des fibules du type Ficarolo. Il est significatif que, à part les fibules, la sépulture d'Otvöspuszta contenait une grande plaqueboucle (fig. 2.11,12) illustrant les éléments du costume germanique oriental (Bierbrauer 1975, Abb. 6.1,2; I Goti 1994, III.7).

\section{LES FIBULES DU TYPE D'UDINE-PLANIS}

(fig. 4.1,2,4).

\section{Souyri (Aveyron).}

Ces fibules (voir à leur propos Bierbrauer 1975, 89-91) se caractérisent par le décor végétal longitudinal symétrique du pied, bordant la ligne verticale médiane, qui sépare le pied en deux champs horizontaux. La tête semi-circulaire porte elle aussi le décor végétal stylisé. Les doigts cantonnant la tête et les protubérances circulaires figurant sur le pied sont parfois ornés des grenats. Le pied se termine par le masque zoomorphe. L'anse est divisée en deux champs par la ligne longitudinale en relief. Enfin, les bordures du pied et de la tête, ainsi que l'anse, portent parfois le décor en petits triangles poinçonnés, appelé « la dent du loup ». Selon V. Bierbrauer les fibules du type Udine-Planis (groupe I des fibules italo-ostrogothiques) appartiennent à la fin du $\mathrm{V}^{\mathrm{e}}$ premier tiers du $\mathrm{VI}^{\mathrm{e}}$ siècles (Bierbrauer 1975, 113). Le mobilier funéraire de la tombe de Lörrach, en Allemagne $\mathrm{du}$ Sud, est très important pour la datation de fibules du type Udine-Planis, car il contenait, à part l'agrafe en question, une épingle à décor aviforme, du type 314 selon la typologie de R. Legoux, P. Périn et F. Vallet, datée de 440/450 à 520/530 (Legoux, Périn, Vallet 2009, $\mathrm{N}^{\circ} 314$ ) et une bague analogue à celle d'une sépulture " princière " à Bakodpuszta, en Hongrie, datée du milieu-troisième quart du Ve siècle (Vallet, Kazanski, De Pirey 1995, 117). D'autre part, une paire des fibules du type Udine-Planis a été mise au jour dans la tombe 270 de la nécropole normande de Saint-Martin-de-Fontenay (fig. 4.4), avec deux fibules cloisonnées (Pilet 1994, pl. 34.1), typiques de l'époque mérovingienne ancienne, surtout de la période allant de 470/480 à 560/570 (Legoux, Périn, Vallet 2009, № 207).

Une fibule dérivée de ce type a été découverte à Souyri (Aveyron, aujourd'hui la commune de Salles-laSources) (fig. 1.2), à en juger d'après le dessin assez schématique et la description de G. Barrière-Flavy (fig. 4.1). Ses doigts sont ornés des grenats (BarrièreFlavy 1892, 140, pl. III.2).

En dehors du Sud-Ouest, les fibules du type d'UdinePlanis ont été mises au jour en Gaule de l'Est, à Brochon (fig. 4.2) (Vallet, Kazanski, de Pirey 1995, 117, fig. 4.1), et au Nord, à Saint-Martin-de-Fontenay (voir supra) (fig.
4.4). Ces fibules, très probablement dérivées du même type d'Udine-Planis sont attestées en Espagne wisigothique, notamment à El Carpio de Tajo, tombe 194 (Ripoll López, 1994, fig. 16) et à Castilltierra, tombe 449 (Arias Sánchez, Balmaseda Muncharaz 2015, 990, 991) (fig. 4.5). Les versions différentes des fibules du type Udine-Planis se diffusent dans les Balkans orientales et en Crimée, dans les zones d'installation des Goths (Kazanski 1996, 330 ; Gavritukhin, Kazanski 2010, 114-116 ; Dumanov 2012, 120-122). D'après les ensembles clos, tels que Kertch-Dolgaia Skala, tombe de 1875, Kertch, tombe 78 de 1907, Kertch 180 de 1904, la date des fibules du type Udine-Planis dans la région pontique englobe tout le VIe siècle (Kazanski 1996, 330 ; Gavritukhin, Kazanski 2010, 100, 101, 116). La diffusion géographique de différentes versions du type UdinePlanis suggère son origine du milieu gothique du royaume d'Italie et des marges pontiques de l'Empire d'Orient. Une paire de fibules venant de Tasov (fig. 4.3), au pays nord-danubien, datée de la fin du Ve-début du $\mathrm{VI}^{\mathrm{e}}$ siècles, représente une version peut-être la plus ancienne de ce groupe de fibules (Tejral 2002, 327, 328, Abb. 9.3,4 ; Tejral 2008, 267, Abb. 12.6).

\section{LES FIBULES DU TYPE D'ESTEBANVELA} (fig. 4.6-8).

\section{Sérignan (Hérault), Saint-Affrique (Aveyron).}

Ce sont les agrafes à cinq doigts et à décor en zigzag longitudinal symétrique sur le pied, de deux côtes de la ligne médiane verticale hachée. La tête porte le décor géométrique. Les bordures du pied et de la tête sont ornées des lignes hachées. Le pied possède quatre protubérances circulaires et se termine par le masque zoomorphe stylisé. Les doigts, les protubérances et le masque portent les grenats. L'anse est divisée en deux champs par une ligne longitudinale en relief. Selon W. Ebel-Zeperzauer c'est un type hispano-wisigothique d'Estebanevela (Ebel-Zeperzauer 2000, 23, 24), représenté en Espagne à Estebanvela et Madrona, tombe 238 (fig. 4.8). À part les fibules citées par W. EbelZeperzauer (4), il faut y ajouter une paire de fibules provenant de la tombe 51 de la nécropole de Hereira de Pisuerga. La sépulture contenait aussi une grande plaque-boucle de ceinture (Morillo Cedrán 1999, Photo 2).

Une pièce de ce type, en bronze, de longueur de $11,7 \mathrm{~cm}$ vient de Sérignan (Hérault) (fig. 1.3 ; 4.6) (Landes 1988, 185, $\mathrm{n}^{\circ}$ 10, Hernandez, Raynaud 2005, fig. 1.B). Une autre fibule, en bronze (fig. 4.7), a été mise au jour à Saint-Affrique (Aveyron) (fig. 1.4), dans la cimetière de la Montagne des Anglais, dans une nécropole avec des tombes en dalles des pierres, disposées selon l'axe Est-Ouest (Cartailhac 1902, pl. 3.2).

\footnotetext{
${ }^{4}$ Mais à mon avis la liste des fibules, citées par W. Ebel-Zeperzauer est hétéroclite, car elle réunie les fibules au décor végétal, comme Estagel (fig. 5.2,3) et à celui géométrique, en zigzag sur le pied (Sérignan, Saint-Affrique, Madrona) (fig. 4.6-8).
} 
Comme parallèle extérieur on peut citer une fibule venant de la collection Diergardt, provenant du Nord de la mer Noire («Kertch » - collections Massonneau et Diergardt) (fig. 4.9). Elle a été attribuée par J. Werner à la forme locale (südrussiche) des fibules d'Udine-Planis (voir supra.) et par conséquent datée de la première moitié du VIº siècle (Werner 1961, 30, Taf. 27, N 110).

Il faut noter que le décor en zigzag sur le pied est attesté relativement tôt sur les fibules, dès le milieu du $\mathrm{V}^{\mathrm{e}}$ siècle, comme sur les exemplaires de Zemun dans la région danubienne (Bierbrauer 1975, Taf. LXXVI.1,2 ; Tejral 1997, 349, Abb. 23.17, 18). Il n'est pas exclu que le décor en zigzag représente la version simplifiée des motifs végétaux des fibules du type Udine-Planis.

\section{LES FIBULES DU TYPE \\ D'ARCY-SAINTE-RESTITUE (fig. 5.1-5).}

\section{Larroque-Castayrols (Tarn),}

Estagel (Pyrénées-Orientales).

Ce sont les fibules à cinq doigts, portant un décor à quatre volutes dans la partie centrale du pied et à deux volutes opposées sur la tête, parfois séparées par un triangle imitant la taille biseautée. L'anse parfois est décorée par plusieurs lignes longitudinales en relief. Le pied est orné de six protubérances circulaires, parfois incrustés de grenats. Le pied se termine par un masque zoomorphe, la bordure de la fibule porte parfois le décor «en dent du loup». Ces fibules, elles aussi, sont dérivées du type danubien de Sokolnice (voir supra). Parmi les prototypes directs on peut citer les fibules provenant d'une tombe à Čukarica (Ostružnica), à Belgrade d'aujourd'hui. Cette sépulture contenait aussi deux fibules en arbalète du type Smolin, datée du milieu du Ve siècle (cf. L'Or des princes barbares 2000, $\mathrm{n}^{\circ} 22.6$; à propos de la tombe princière de Smolin voir en détail : Tejral 1973, 25-53), deux petites copies du même type en arbalète, un collier des perles et deux boucles d'oreille à polyèdre vide (Tatić-Curić 1958; Bierbrauer 1975, Taf. 78) (fig. 2.6-10).

Une fibule du type d'Arcy-Sainte-Restitue, assez dégradée, provient de la nécropole de LarroqueCastayrols (Tarn) (fig. 1.5). Elle a été découverte durant la prospection du champ où auparavant les agriculteurs avaient mis au jour une grande plaque-boucle à plaque rectangulaire de type " wisigothique " et une perle en ambre (Cubaynes, Lasserre 1966, 310, pl. 98). La fibule est en alliage cuivreux, à surface dorée, avec les restes d'ardillon en fer (5) (fig. 5.1).

Une autre fibule dérivée de ce type a été mise au jour en 1869 à Estagel (Pyrénées-Orientales) (fig. 1.6), dans une tombe, sur la poitrine du défunt. Elle est ornée des grenats (Barrière-Flavy 1892, 134, pl. III.1) (fig. 5.3).
Une autre pièce similaire provient de la tombe 8 de la même nécropole en 1935 (Lantier 1943, 158, fig. 3. T.8) (fig. 5.2). Cette fibule est en bronze, de la longueur de $10,1 \mathrm{~cm}$ et porte le décor en deux volutes sur la tête et en quatre volutes sur le pied (Landes 1988, n ${ }^{\circ}$ 17; I Goti 1994, fig. IV.42). Elle est attribuée par W. EbelZeperzauer au type Estebanvela (voir infra), cependant le décor du pied est différent - il comporte les éléments végétaux (Ebel-Zeperzauer 2000, 23).

Mais deux exemplaires les plus travaillés de ce type viennent de la Gaule du Nord, de la nécropole d'ArcySainte-Restitue, tombe 1727 (Aisne) (fig. 5.4). Elles sont en alliage cuivreux, de 10,4 cm de longueur (Koch 1998, 601, N ${ }^{\circ} 144$, Taf. 35.4,5) (6). D'autre part un pied de la fibule, très vraisemblablement appartenant au même type Arcy-Sainte-Restitue, vient de la Seine-Maritime (Kühn 1998, N 618, 660, Taf. 35.5 ; Lorren 2001, pl. II.2) (fig. 5.5). En ce qui concerne leur date, ces fibules, compte tenu leur ressemblance morphologique avec les agrafes du type Envermeu (voir infra), doivent avoir à peu près la même chronologie, c'est-à-dire le dernier tiers du Ve-premier tiers du VIe siècles.

\section{LES FIBULES DU TYPE D'ENVERMEU}

(fig. 5.7-9,11).

\section{Toulouse, Saint-Affrique (Aveyron), Monteils (Tarn-et-Garonne).}

Il s'agit des fibules qui ressemblent au type d'ArcySainte-Restitue, mais leur décor est différent. Elles portent le décor radial sur la tête et celui quadrillé sur le pied (Koch 1998, 236-238, Taf. 35.6, Karte 18). Dans un cas (Monteils, voir infra.), la tête des agrafes est décorée de deux têtes aviformes ornées des grenats. L'anse est divisée en deux champs par une ligne longitudinale en relief. Ces fibules ont été mises au jour dans le SudOuest de la Gaule, donc dans la zone wisigothique, à Toulouse (Barrière-Flavy 1892, pl. 3.2) (fig. $1.7 ; 5.9$ ), ainsi qu'à Saint-Affrique (Aveyron), dans le cimetière de la Montagne des Anglais, déjà citée (Cartailhac 1902, pl. 3.1). Cette fibule est en bronze (fig. 5.8). Enfin deux fibules de ce type sont découvertes dans la nécropole de Monteils (Tarn-et-Garonne) (fig. 1.8). Il s'agit d'une tombe contenant aussi deux boucles d'oreille à pendentif polyédrique, près du crâne et une plaque-boucle de ceinture, ornée de grenats (Lapart, Neveu 1987, 50, pl. 1) . Les fibules sont en alliage cuivreux, à surface dorée, de $15,5 \mathrm{~cm}$ de longueur, ornées des pierres ou des verres rouges (fig. 5.11). Ce type est également attesté en Espagne wisigothique (Duratón ou Castiltierra) (I Goti 1994, fig. IV.5), mais ici les fibules portent sur la tête un décor végétal (fig. 5.6). D'autre part les fibules à décor quadrillé sur le pied et celui en volutes sur la tête sont

\footnotetext{
${ }^{5}$ La fibule est publiée sans échelle et sa taille n'est pas précisée dans le texte de la publication de 1966.

${ }^{6}$ On pourrait supposer donc, que ce type de fibules, assurément de la tradition germanique orientale, se forme d'abord en Gaule du Nord, ensuite ces dérivées arrivent en Gaule du Sud - Ouest, sur le territoire wisigothique. Cependant le nombre très limité des découvertes ne nous permet pas de confirmer ou infirmer une telle supposition.
} 
connues en Italie, notamment à Tortona (Bierbrauer 1975, Taf. XLIV.1,2, LXIV.1,2). Mais d'autre part les fibules italiennes, d'après leurs proportions sont plus proches au type Mistrín (voir infra.).

En dehors de la zone wisigothique les fibules de ce type ont été mises au jour à Envermeu (Seine-Maritime), dans une tombe, découverte en 1850 (Flavigny 1975, $n^{\circ}$ 632; Koch 1998, 622, N² 297, Taf. 35.6; Lorren 2001, pl. 2.3). C'est une paire de fibules en bronze, plaquée d'argent, de la longueur de 10,6 cm (fig. 5.7). Si les informations sur la découverte d'Envermeu sont exactes (Flavigny 1975, 153, 154), la fibule de la tombe de 1850 a été mise au jour avec un bracelet du type mérovingien 337 , des pinces à épiler du type 320, une épingle apparentée au type 314, les rivets du type 195 et une boucle de ceinture du type 110 (selon Legoux, Périn, Vallet 2009). La chronologie de ces objets correspond à la période mérovingienne ancienne 1 (470/480 à 520/530).

Comme parallèle danubien lointain il faut citer une paire des fibules provenant de la tombe 56 de la nécropole gépide de Szentes-Kökenzug (Csallány 1961, Taf. XI.1,2). Ces fibules ont sur le pied seulement quatre protuberances au lieu de six (fig. 5.10). Il faut souligner la ressemblance morphologique des fibules appartenant aux types d'Arcy-Sainte-Restitue et d'Envermeu, ce que témoinge à la faveur de leurs origines communes.

\section{LES FIBULES DU TYPE DE MISTŘÍN (fig. 6).}

Les fibules de ce type (typologie selon Tejral 2002, 321-326, Abb. 7), à cinq ou à sept doigts, possèdent le décor quadrillée sur le pied et celui à deux volutes opposées sur la tête, parfois séparées par un triangle en taille biseautée. Le pied se termine par le masque zoomorphe. L'anse est divisée en deux champs par une ligne longitudinale en relief. Deux fibules du type Mistrín ont été découvertes dans une sépulture près de l'église de SaintPierre-des-Cuisines, à Toulouse (fig. 1.7). Ici, au chevet de l'église, on a mis au jour deux inhumations « habillées » (Lequément 1986, fig. 15). La première tombe contenait une grande plaque-boucle habituelle pour la zone du royaume wisigothique, à plaque rectangulaire, revêtue de feuille d'argent (voir à son propos Stutz 2000, 35-37). Une autre inhumation, en pleine terre, a livré deux fibules digitées en bronze, de 8,6 et $8,7 \mathrm{~cm}$ de longueur (fig. 6.1). Ces dernières ont été à juste titre identifiées comme appartenant au type danubien de Mistř́ńn (Stutz 2000, note 23; Bach et al. 2002, 535, fig. 215.16).

En Gaule on connaît encore deux fibules représentant les dérivées de ce type. La première, en argent doré, de $7,7 \mathrm{~cm}$ de longueur, provient de Fère-en-Tardenois
(Aisne) (Caillet 1985, n 96) (fig. 6.2), l'autre, avec sept doigts, en argent doré, de la longueur de $7,7 \mathrm{~cm}$, provient de la nécropole mérovingienne d'Arcy-Sainte-Restitue, tombe 2408 (Koch 1998, 603, $\mathrm{N}^{\circ}$ 154, Taf. 36.8; Tejral 2002, 322) (fig. 6.3)

Les fibules du type de Mistrín sont bien attestées dans les pays nord-danubiens de l'époque « prélombarde » (avant l'arrivée des Lombards sur le Danube moyen vers 512 ), c'est-à-dire de la deuxième moitié du Ve siècle, plutôt des dernières décennies du $\mathrm{V}^{\mathrm{e}}$ siècle. On peut citer les découvertes à Mistřín (fig. 6.4), à Schletz, tombe 10 (fig. 6.5), à Vienne-Salvator Gasse (fig. 6.8), et, en dehors de la région au Nord du Danube, à Altenerding, tombes 272 et 554, en Bavière (fig. 6.7). Les découvertes à Schletz et à Vienne-Salvator Gasse montrent que ces fibules ont été portées selon la mode féminine des Germains orientaux, sur les épaules (Martin 2002, 214, Abb. 14; Tejral 2002, 321-326, Abb. 7). En même temps ces agrafes ont été mises au jour en Hongrie orientale, à l'est du Danube, c'est-à-dire sur le territoire du royaume gépide : à Bókeny-Mindszent (Hampel 1905, Taf. 56.10), à Magyartés (fig. 6.9), Hódmezővásárhely-Gorsza (Csallány 1961, Taf. CIX.1-3 ; CCXXXIII.8,12) et à Tarnamera (Bóna, Nagy 2002, Taf. 56.1) (fig. 6.6). Les dérivées du type Mistř́n sont également connues en Italie (Bierbrauer 1975, Taf. LXXXII.3) (7).

Les fibules de tradition danubienne représentent le témoignage archéologique des contacts entre la Gaule du Sud et les royaumes germaniques orientaux du Danube moyen et d'Italie, au même titre que la plaque-boucle gépide à tête d'aigle et les petites fibules ansées, découvertes en Gaule méridionale (Kazanski 2010 ; Kazanski 2013). Il faut souligner que ces contacts, à part les relations avec l'Italie ostrogothique, sont peu attestés par les auteurs anciens. Cela n'est pas étonnant, car le matériel archéologique et les sources écrites montrent l'histoire d'une façon différente. C'est d'autant plus exact pour les « classes moyennes » des anciennes sociétés, car les témoignages écrits de la fin de l'Antiquité et du début du Moyen Âge, on le sait, concernent avant tout les élites dirigeantes et guerrières et les faits d'histoire politique, militaire et dynastique. Or, les fibules digitées de petite et moyenne taille chez les Germains orientaux sont avant tout l'apanage du costume féminin des « classes moyennes » (Ambroz 1968, 22 ; Bierbrauer 1989, 152-155 ; Kazanski 2013, 55, 56). Compte tenu le caractère " ethnographique " du costume féminin dans les sociétés traditionnelles (Périn, Kazanski 2006, 191, 192), on peut conclure que l'apparition de ces modèles est liée avec la migration de petits groupes ou et même les personnes isolées d'origine (ou de tradition culturelle ?) germanique orientale.

\footnotetext{
Il existe en Gaule, plus au Nord, un autre type des fibules, dit Hahnheim, qui est également dérivée de celles de Mistřín, répandu en Gaule du Nord, sur l'île britannique et en Germanie. Les fibules du type Hahnheim se distinguent par le décor de tête plus dégradé, en forme de trois triangles. Ce type est daté de la première moitié-milieu du VIe siècle (Koch 1998, 203-205, Karte 16, Taf. 29.5-10, 30, 31.1-5; Kazanski 2002, 21, 22). Enfin, encore un groupe des fibules digitées, qui remonte peut-être au même prototype Mistř́n, est celui de Cutry/Naumburg/Kolleda, attesté sporadiquement en Gaule du Nord, notamment à Cutry, tombe 919 et à Monceau-le-Neuf-en-Faucouzy (Koch 1998, Taf. 28.2,3), mais surtout en Thuringe (Koch 1998 , 188-191, Karte 15 ; Bemmann 2008, 184, Abb. 38)
} 

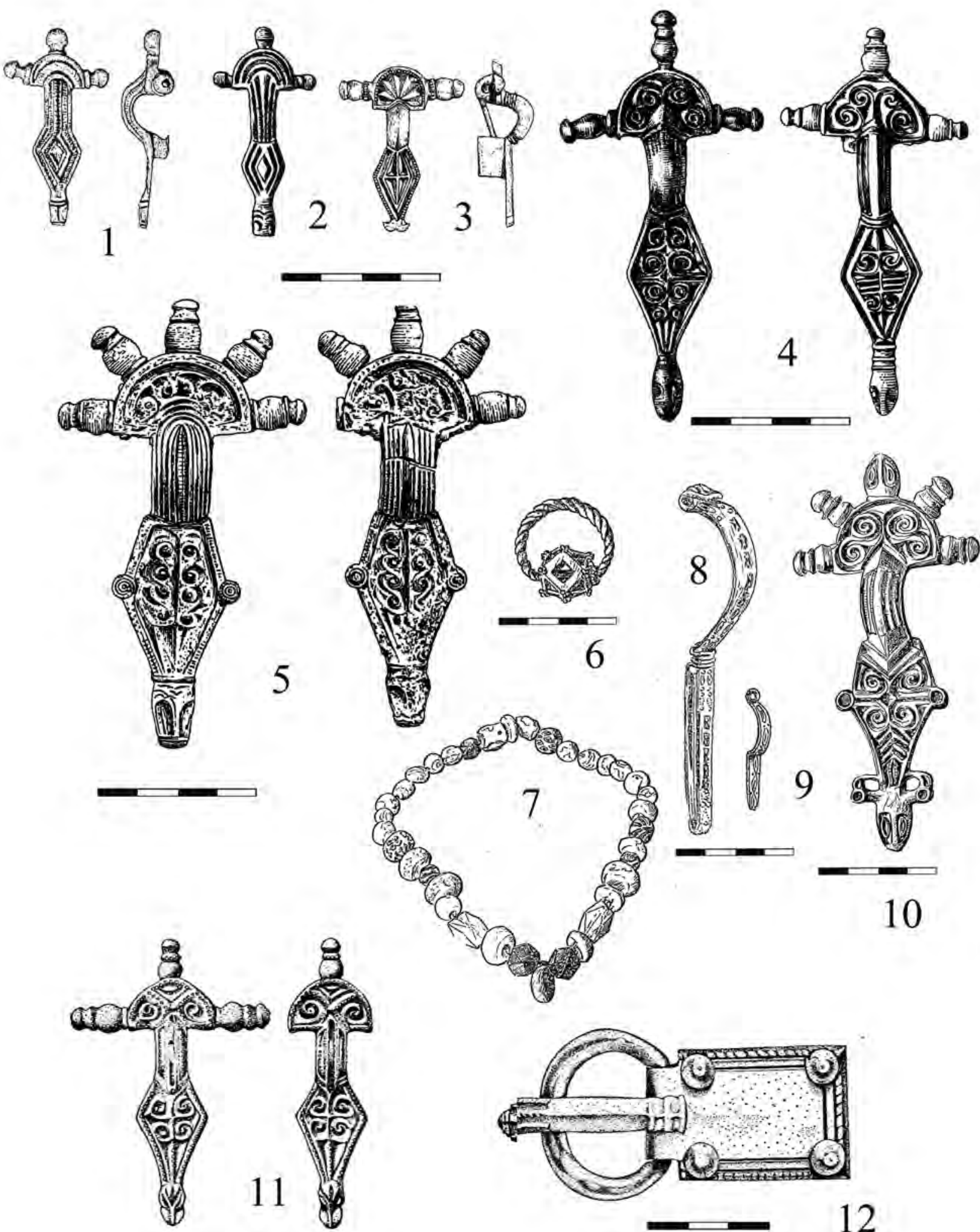

Fig. 2 : Les fibules digitées danubiennes du milieu -deuxième moitié du $\mathbf{V}^{\mathbf{e}}$ siècle. 1 : Cifer-Pác ; 2 , 4 : Sokolnice ; 3 : Bernhardsthal-Aulüssen; 5 : Tasov ; 6-10 : Belgrade-Ostružnica ; 11-12: Dabronc-Ötvöspuszta. 1-5 : d'après Tejral 2002, Abb. 6.1,2,7-9 ; 6-10 : d'après Татић-Ђурић 1958, sl. 1-4;11,12 : d'après Bierbrauer 1975, Abb. 6.7 : sans échelle. 

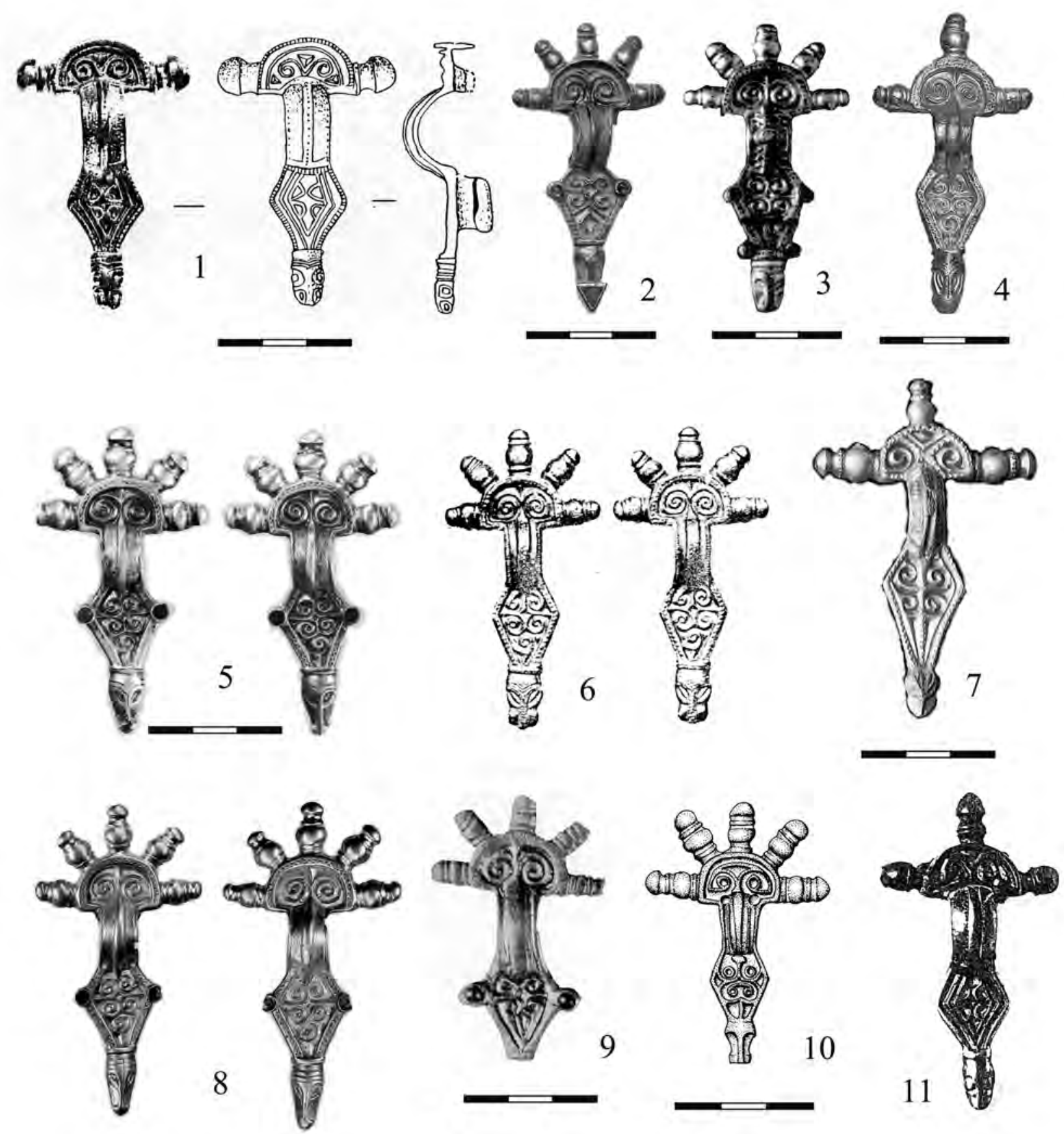

Fig. 3 : Les fibules du type Ficarolo et leur prototype possible (7). 1 : Taradeau-Saint-Martin ; 2 : Brochon ; 3 : Chaussin ; 4 : Ficarolo ; 5 : Bâle-Kleinhüningen, tombe $75 ; 6$ : Bâle-Gotterbarmweg, tombe $22 ; 7$ : Dabronc-Ötvöspuszta ; 8 : CsongrádKettoshalöm ; 9 : Stössen, tombe 69 ; 10 : Bojná ; 11 : Stehlčeves. 1 : d'après Boyer 1971, 153, 154, fig. 6.3 ; 2: d'après Vallet, Kazanski, De Pirey 1995, fig. 4.5; 3 : d'après Koch 1998, Taf. 34.2 ; 4: d'après I Goti 1994, fig. III.61; 5 : d'après GieslerMüller 1992, Taf. 67.3,4 ; 6 : d'après Vogt 1930, Taf. 10.XXII.3,4; 7 : d'après I Goti 1994, fig. III.27 ; 8 : d'après Csallány 1961, Taf. CCXI.14,15; 9 : d'après Bierbrauer 1975, Taf. 70.5 ; 10 : d'après Pieta 2006, obr. 3.1 ; 11 : d'après Svoboda 1975, obr. 10. 6,8,11: sans échelle. 

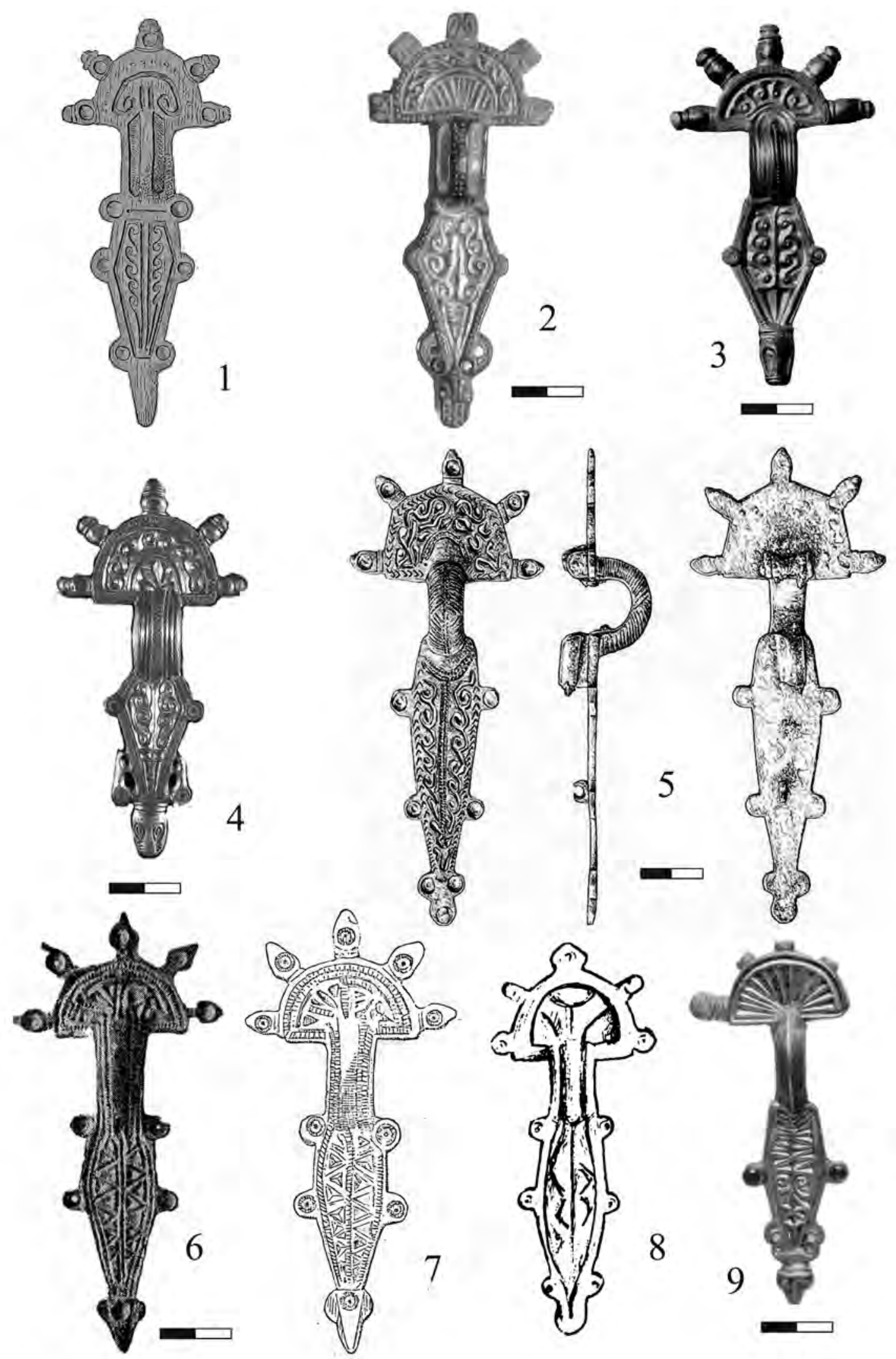

5
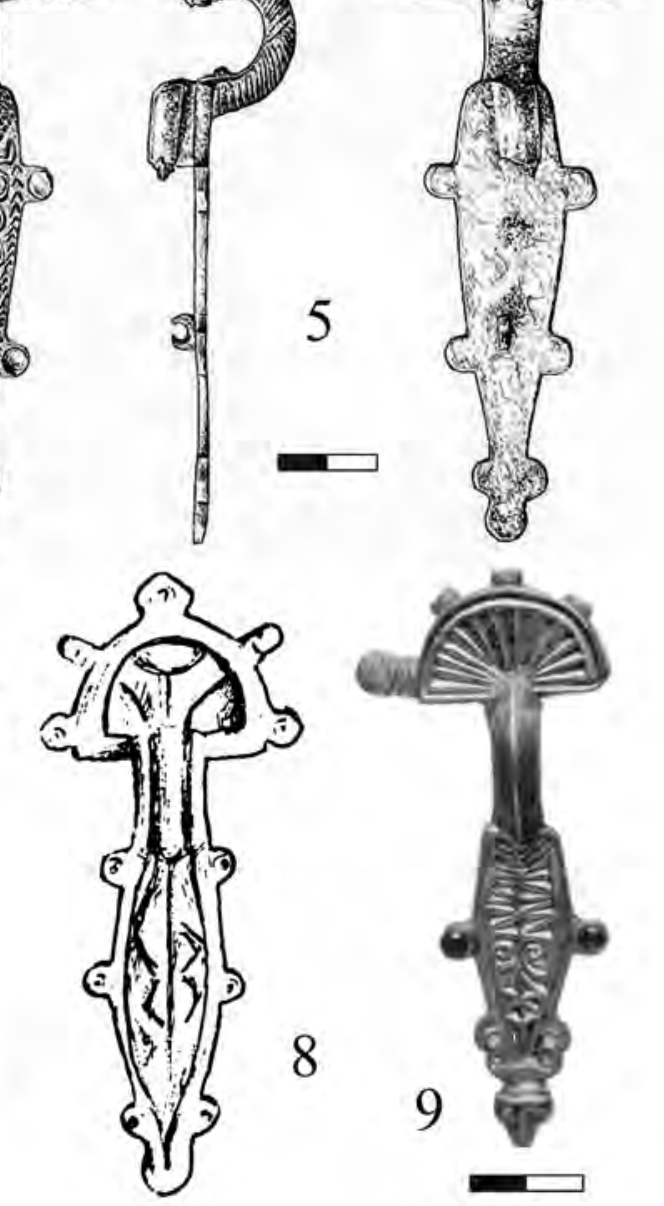

Fig. 4 : Les fibules du type Udine-Planis $(\mathbf{1 , 2 , 4 )}$, leurs prototypes possibles (3), leurs dérivées (5) et les fibules du type Estabanvela (6-9). 1 : Souyri ; 2 : Brochon ; 3 : Tasov; 4 : Saint-Martin-de-Fontenay, tombe $270 ; 5$ : Castiltierra, tombe $449 ; 6$ : Sauvian ; 7 : Saint-Affrique ; 8 : Madrona, tombe $238 ; 9$ : «Kertch », collections Massoneau-Diergardt. 1 : d'après Barrière-Flavy 1892, pl. III. 2 ; 2 : d'après Vallet, Kazanski, De Pirey 1995, 117, $4.1 ; 3$ : d'après Bierbrauer 1975, Taf. 75.1; 4 : d'après Pilet 1994, fig. 51 ; 5 : d'après Arias Sánchez, Balmaseda Muncharaz 2015, 990, 991 ; 6 : d'après Hernandez, Raynaud 2005, fig. 1.B; 7 : d'après Cartailhac 1902, pl. 3.2; 8 : d'après Ebel-Zeperzauer 2000, Taf. 19.44; 9 : d'après Werner 1961, 30, Taf. 27, $\mathrm{N}^{\circ}$ 110. 1, 7, $8:$ sans échelle. 

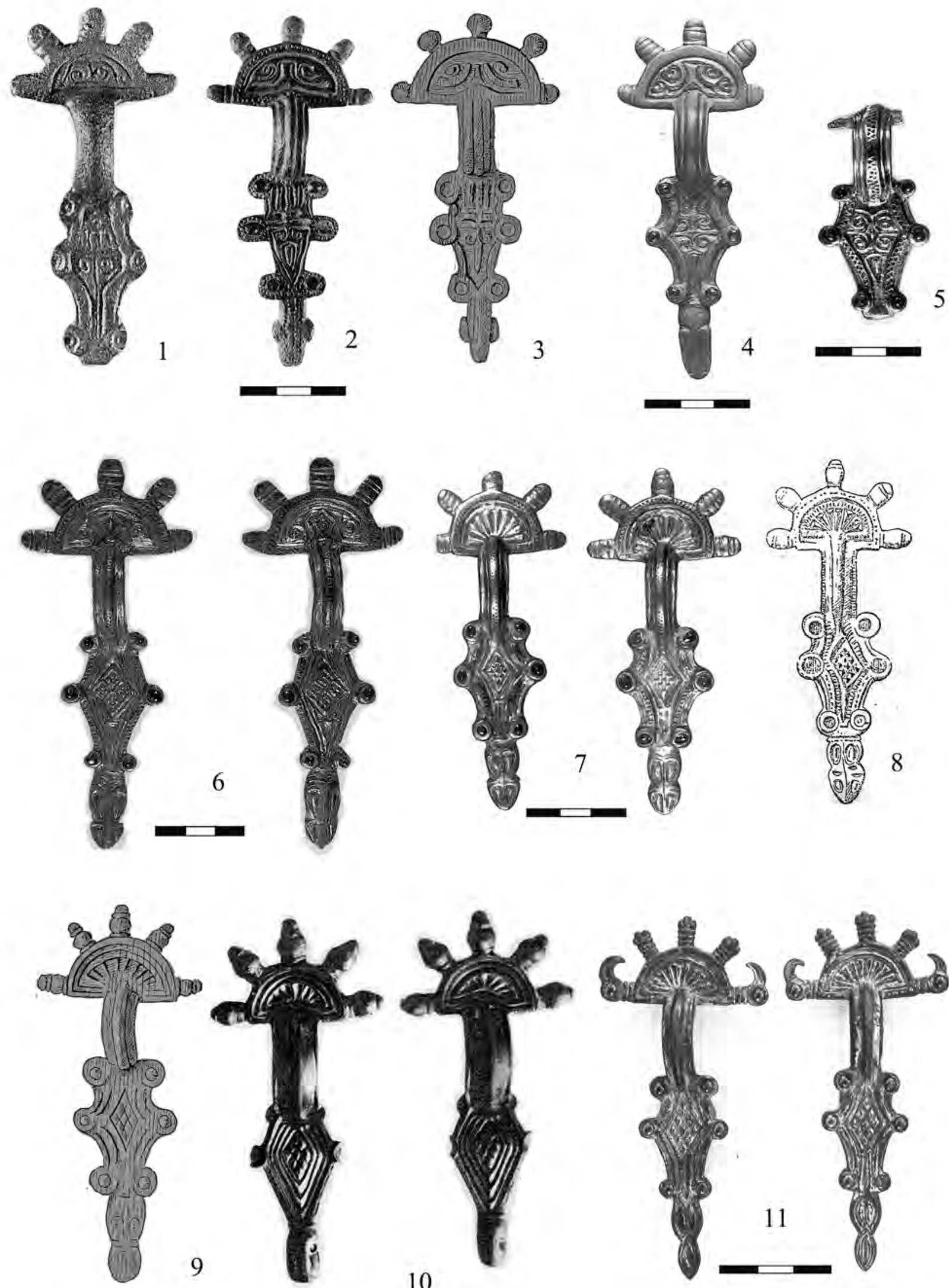

Fig. 5 : Les fibules des types Arcy-Sainte-Restitue (1-5) et Envermeu (7-9,11) et leurs parallèles (6, 10). 1 : Larroque-Castayrols; 2 : Estagel, tombe 8; 3 : Estagel, tombe de 1869; 4 : Arcy-Sainte-Restitue, tombe $1727 ; 5$ : Seine-Maritime ; 6 : Duratón ou Castiltierra ; 7 : Envermeu ; 8 : Saint-Affrique ; 9 : Toulous e; 10 : Szentes-Kökenzug ; $11:$ Monteils . $1:$ d'après Cubaynes, Lasserre 1966, pl. 98 ; 2 : d'après Lantier 1943, fig. 3. T.8; 3 : d'après Barrière-Flavy 1892, pl. III.1; 4 : d'après Koch 1998, Taf. 35.4,5; 5 : d'après Lorren 2001, pl. II.2; 6 : d'après I Goti 1994, fig. IV.5; 7 : d'après Lorren 2001, pl. 2.3; 8 : d'après Cartailhac 1902, pl. 3.1; 9 : d'après Barrière-Flavy 1892, pl. 3.2; 10 : d'après Csallány 1961, Taf. XI.1,2; 11 : d'après Lapart, Neveu 1987, pl. 1. 1, 3, 8, 9, 10 : sans échelle. 

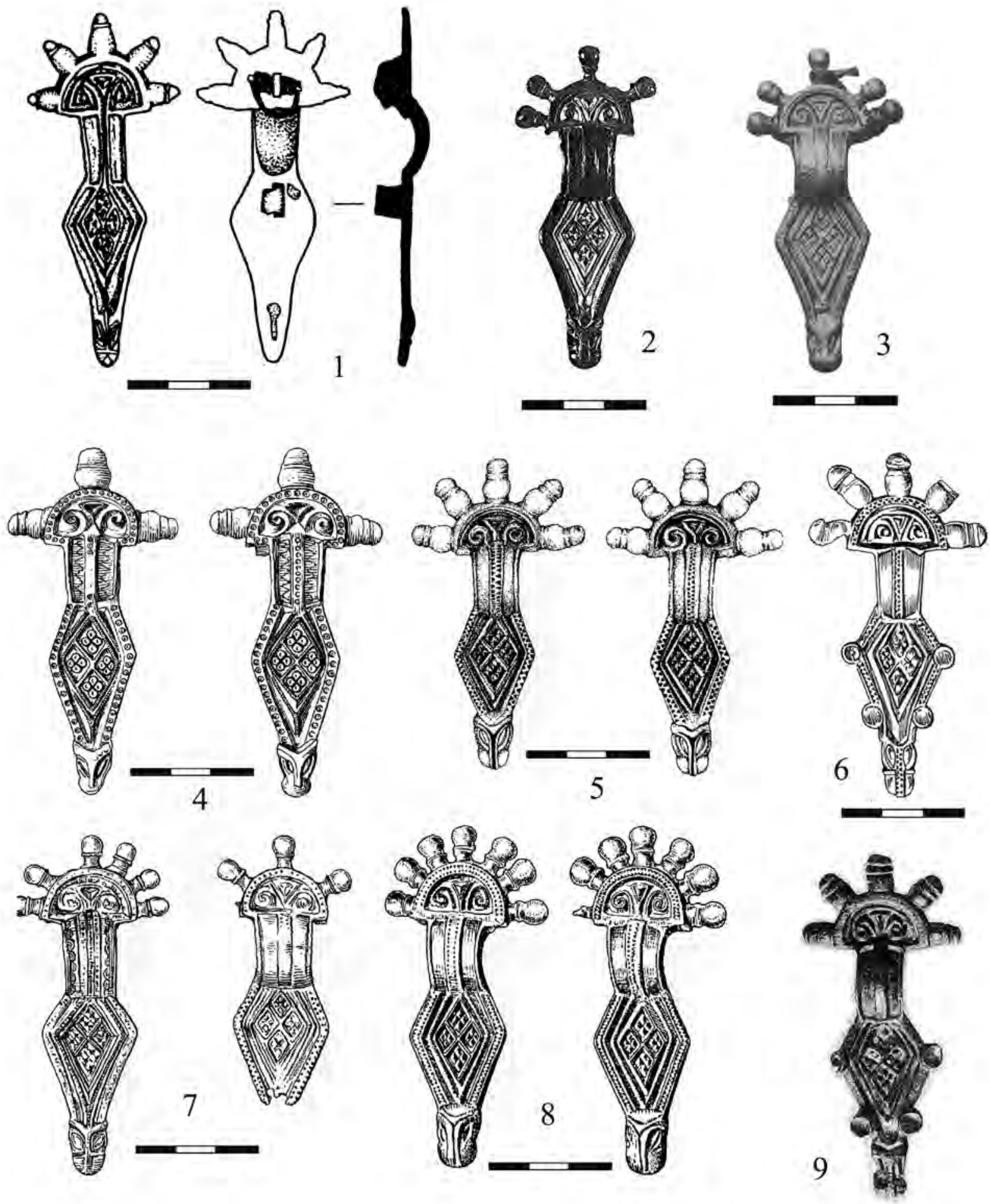

Fig. 6 : Les fibules du type Mistř́n. 1 : Saint-Pierre-des-Cuisines, Toulouse ; 2 : Fère-en-Tardenois; 3 : Arcy-Sainte-Restitue, tombe $2408 ; 4:$ Mistř́n ; 5 : Schletz, tombe 10; 6 : Tarnamera ; $7:$ Altenerding, tombes 554 et $272 ; 8$ : Vienne-Salvatorgasse, tombe S2 ; 9 : Magyartés. 1 : d'après Bach et alii 2002, 535, fig. 215.16; 2 : d'après Caillet 1985, n 96; 3 : d'après Koch 1998, Taf. 36.8; 4,5,7,8 : d'après Tejral 2002, Abb. 7; 6 : d'après Bóna, Nagy 2002, Taf. 56.1; 9 :d'après Csallány 1961, Taf. CIX. 2.9 : sans échelle. 


\section{BIBLIOGRAPHIE}

Ambroz 1968 : АМБРОЗ (А.К.), Дунайские элементы в раннесредневековой культуре Крыма (VI-VII вв.). Краткие Сообшения Института Археологии, $\mathrm{N}^{\circ} 113,1968$, p. 10-23.

Arias Sánchez, Balmaseda Muncharaz 2015 : ARIAS SÁNCHEZ (I.), BALMASEDA MUNCHARAZ (L.J.), La necrópolis de época visigoda de Castiltierra (Segovia) Excavaciones dirigidas por E. Camps y J. M. ${ }^{a}$ de Navascués, 1932-1935. Materiales conservados en el Museo Arqueológico Nacional. Madrid / Museo Arqueológico Nacional, 2015, 1130 p.

Bach et al. 2002 : BACH (S.), BOUDARTCHOUK (J.L.), CAZES (J.-P.), RIFA (P.), STUTZ (F.), Le mobilier funéraire, témoin d'influences culturelles et d'une possible présence germaniques, in : PAILLER (J.-M.), éd., Tolosa. Nouvelles recherches sur Toulouse et son territoire dans l'Antiquité. Rome / Ecole française de Rome, 2002, p. 533-543.

Barrière-Flavy 1892 : BARRIÈRE-FLAVY (G.), Étude sur les sépultures barbares du Midi et de l'Ouest de la France. Industrie wisigothique. Toulouse-Paris / P. Ed. Privat, E. Leroux, 1892, 240 p.

Bemmann 2008 : BEMMANN (J.), Mitteldeutschland im 5. Jahrhèndert -Eine Zwischenstation auf dem Weg der Langobarden in den mittleren Donauraum?, in: BEMMANNN (J.), SCHMAUDER (M.), éd., Kulturwandel in Mitteleuropa. LangobardenAwaren-Slawen. Bonn / Dr. Rudolf Habelt GhbH, 2008, p. 145-227.

Bierbrauer 1975 : BIERBRAUER (V.), Die ostgotischen Grab- und Schatzfunde in Itlaien. Spolète / Centro Italiano di Studi sull'Alto Medioevo, 1975, 378 p.

Bierbrauer 1989 : BIERBRAUER (V.), Bronzene Bügelfibeln des 5. Jahrhunderts aus Südosteuropa. Jahresschrift für Mitteldeutsche Vorgeschichte, vol. 72, 1989, p. 141-160.

Bierbrauer et al. 1993 : BIERBRAUER (V.), BÜSING (V.), BÜSING (H.), Die Dame von Ficarolo. Archeologia Medievale, vol. 20, 1993, p. 303-332.

Bóna, Nagy 2002 : BÓNA (I.), NAGY (M.), Gepidische Gräberfelder am Theissgebiet I. Budapest /Magyar Nemzeti Múzeum, 2002, 388 p.

Boyer 1971 : BOYER (R.), Pièces de mobilier funéraire du Haut Moyen Àge découvertes dans le Var. Revue des études ligures, vol. 37, 1971, p. 148-154.

Caillet 1985 : CAILLET (J.-P.), L'antiquité tardive, le haut moyen age et Byzance au Musée Cluny. Paris / Réunion des musées nationaux, 1985, 272 p.

Cartailhac 1902 : CARTAILHAC (E.), Le cimetière barbare de Saint-Affrique (Aveyron). Bulletin de la Société Archéologique du Midi, vol. 29/IV, 1902, p. $35-37$

Cazes 1987 : CAZES (Q.), Toulouse, Saint-Pierre-desCuisines, in : Dix ans de recherches archéologiques en Midi-Pyrénées. Toulouse / Musée Saint-Raymond, 1987, p. 145-147.
Csállany 1961 : CSÁLLANY (G.), Archäologische Denkmäler der Gepiden in Mitteldonaubecken (454-568 u. Z.). Budapest: Verlag der Ungarischen Akademie der Wissenschaften, 1961, 406 p.

Cubaynes, Lasserre 1966 : CUBAYNES (R.), LASSERRE (F.), Le cimetière wisigothique de Larroque-Gestayrols (Tarn). Ogam, vol. 18, 1966, p. $305-310$

Dumanov 2012 : ДУМАНОВ (Б.), Долнодунавските готски паметнице, украсени с kerbschnitt. Фибули. Селищна Археология, vol. VII, 2012, p. 112-151.

Ebel-Zeperzauer 2000 : EBEL-ZEPERZAUER (W.), Studien zur Archäologie der Westgoten von 5.-7. Jh. n. Chr. Mainz / Phillipp von Zabern, 2000, 408 p.

Flavigny 1975 : FLAVIGNY (L.), L'abbé Cochet et l'archéologie mérovingienne, in : La Normandie souterraine. II. L'abbé Cochet archéologue. Rouen / Musée départemental des antiquités, 1975, p. 135-190.

Gavritukhin, Kazanski 2010 : GAVRITUKHIN (I.), KAZANSKI (M.), Bosporus, the Tetraxite Goths and the Northern Caucasus Region during the Second Half of the Fifth and the Sixth Centuries, in: Curta (F.), éd., Neglected Barbarians. Turnhout /Brepols, 2010, p. 83-136.

Giesler-Müller 1992 : GIESLER-MÜLLER (U.), Das frühmittelalterliche Gräberfeld von BaselKleinhüningen. Derendingen-Solothurn / Habegger Verlag, 1992, 336 p.

I Goti 1994 : I Goti. Milan / Electa Lombardia, 1994, 400 p.

Hampel 1905 : HAMPEL (J.), Alterthümer des Frühen Mittelalters in Ungarn. Bd. III. Braunshweig / Verlag von Friedrich Vieweg und Sohn, 1905, 904 p.

Hernandez, Raynaud 2005 : HERNANDEZ (J.), RAYNAUD (C.), La Septimanie du $\mathrm{V}^{\mathrm{e}} \mathrm{au}$ VIII ${ }^{\mathrm{e}}$ siècle : archéologie du changement culturel, in : DELESTRE (X.), PERIN (P.), KAZANSKI (M.), éd., La Méditerranée et le monde mérovingien. Aixen-Provence / Association Provence Archéologie, 2005, p. $177-187$

Kazanski 1996 : KAZANSKI (M.), Les Germains orientaux au Nord de la mer Noire pendant la seconde moitié du $\mathrm{V}^{\mathrm{e}} \mathrm{s}$. et au $\mathrm{VI}^{\mathrm{e}}$ s. Mатериаль no Археологии, Истори и Этнографии Таврии, vol. 5, 1996, p. 324-337, 567-581.

Kazanski 1997 : KAZANSKI (M.), La Gaule et le Danube à l'époque des Grandes Migrations, in: TEJRAL(J.), FRIESINGER (H.), KAZANSKI (M.), éd., Neue Beiträge zur Erforschung der Spätantike im mittleren Donauraum. Brno / Archeologický Ústav Akademie Věd České Republiky, 1997, p. 285-319.

Kazanski 2002 : KAZANSKI (M.), La nécropole galloromaine et mérovingienne de Breny (Aisne). D'après les collections et les archives du Musée des Antiquités Nationales (Europe médiévale, 4). Montagnac / Editions Monique Mergoil, 2002, 270 p. 
Kazanski 2010 : KAZANSKI (M.), Les Gépides en Gaule, in : MĂGUREANU (A.), GÁLL (E.), éd., Intre stepă şi Imperiu. Studii în onoarea lui Radu Harhoiu. Bucureşti / Renaissance, 2010 (2011), p. $127-140$.

Kazanski 2013 : KAZANSKI (M.), Les petites fibules ansées de tradition germanique en Gaule méridionale au Ve siècle. Bulletin de liaison de l'Association française d'archéologie mérovingienne, $\mathrm{N}^{\circ} 34,2013$, p. 54-59.

Köch 1998 : KÖCH (A.), Bügelfibeln der Merowingerzeit im westlichen Frankenreich. Mainz / Verlag der Römisch-Germanischen Zentralmuseums, $1998,750 \mathrm{p}$.

Landes 1988 : LANDES (C.), éd., Gaule mérovingienne et monde méditerranéen. Les derniers Romains en Septimanie, $I V^{e}$-VIII ${ }^{e}$ siècles. Lattes / Imago, 1988., $234 \mathrm{p}$.

Lantier 1943 : LANTIER (M.R.), Le cimetière wisigothique d'Estagel (Fouilles de 1935 et 1936). Gallia, vol. $1 / 1,1943$, p. $153-188$.

Lapart, Neveu 1987 : LAPART (J.), NEVEU (J.), Objets mérovingiens de Monteils près de Caussade (Tarn-et-Garonne), in : Montauban et les anciens pays de Tarn-et-Garonne. Actes du XLI Congrès d'études Régionales. Montauban / Société archéologique de Tarn et Garonne, 1987, p. 49-56.

Legoux, Périn, Vallet 2009 : LEGOUX (R.), PÉRIN (P.), VALLET (F.), Chronologie normalisée du mobilier funéraire mérovingien entre Manche et Lorraine. Saint-Germain-en-Laye / Association française d'archéologie mérovingienne, 2009, 66 p.

Lequément 1986 : LEQUÉMENT (R.), Midi-Pyrénées. Toulouse. Saint-Pierre-des-Cuisines. Gallia, vol. 44/2, 1986, p. 321.

Lorren 2001 : LORREN (C.), Fibules et plaquesboucles à l'époque mérovingienne en Normandie. Paris / Association française d'archéologie mérovingienne, 2001, $554 \mathrm{p}$.

Martin 2002 : MARTIN (M.), „Mixti Alamannis Suevi"? Der Beitrag der alamannischen Gräberfelder am Basler Rheinknie, in : TEJRAL (J.), éd., Probleme der frühen Merowingerzeit im Mitteldonauraum. Brno / Archeologický Ustav Akademie Věd České Republiky, 2002, p. 195-223.

Menke 1986 : MENKE (M.), Archäologische Befunde zu Ostgoten des 5. Jahrhunderts in der zone nordwärts der Alpen, in: KMIECIŃSKI (J.), éd., Peregrinatio Gothica (Archaeologia Baltica VII). Lódz / Katerdra archeologii Uniwersitetu Lódzskiego, 1986, p. 239-282.

Morillo Cedrán 1999 : MORILLO CEDRÁN (A.), Nueva aproximación a los ajuares metálleicos de la necrópolis visigoda de Herrera de Pisuerga (Palencia), in : I Curso de cultura medieval. Actas. Aguilas de Campeo, 1999, p. 233-251.

L'Or des princes barbares 2000 : L'Or des princes barbares. Du Caucase à la Gaule Ve siècle après J.C. Paris / Réunion des Musées nationaux, 2000, $224 \mathrm{p}$.
Périn, Kazanski 2006 : PÉRIN (P.), KAZANSKI (M.), Les tombes féminines à costume « étranger» dans les nécropoles mérovingiennes de Gaule, in : LÓPEZ QUIROGA (J.), MARTÍNEZ TEJERA (A.M.), MORÍN DE PABLOS (J.), éd., Gallia e Hispania en el contexto de la presencia 'germánica' (ss.V-VII). Balance y Perspectivas. Actas de la Mesa Redonda hispano-francesca celebrada en la Universidad Autónoma de Madrid (UAM) y Museo Arqueológico Regional de la Comunidad de Madrid. Oxford / BAR, 2006, p. 191-212.

Pieta 2006 : PIETA (K.), Hradiská Bojná II a Bojná III, in : Pieta K., Ruttkay A., Ruttkay M. (eds), Bojná. Hospodárske a politické centrum Nitranskeho kniažatstva. Nitra / Archeologický ústav SAV, 2006, p. 173-190.

Pilet 1004 : PILET (C.), éd., La nécropole de Saint-Martinde-Fontenay (Calvados). Paris / CNRS Editions, 1994, $552 \mathrm{p}$.

Ripoll López 1994 : RIPOLL LÓPEZ (G.), La necrópolis visigoda de El Carpio del Tajo. Una nueva lectura a partir de la topocronología y los adornos personales. Butlletín de la Reial Académia Catalana de Belles Arts de Sant Jordi, vol. VII-VIII, 1994, p. $187-250$.

von Rummel 2007 : VON RUMMEL (P.), Habitus barbarus. Kleidung und Repräsentation spätantiker Eliten im 4. und 5. Jahrhundert. Belin-New York / Wlater de Gruyter, 2007, 482 p.

Stutz 2000 : STUTZ (F.), L'inhumation habillée à l'époque mérovingienne au Sud de la Loire. Mémoires de la société archéologique du Midi de la France, vol. 60, 2000, p. 33-47.

Svoboda 1975 : SVOBODA (B.), Dva hroby z doby stěhování národů ve Stehelčevsi u Slaného. Památky archeologické 116 (1), vol. 1975, p. 133-151.

Tatić-Curić 1958 : ТАТИН-ЂУРИН (М.), Готски гроб из Остружнице. Зборник Радова Народног музеја у Београдy, vol. 1, 1958, p. 161-185.

Tejral 1973 : TEJRAL (J.), Mähren im 5. Jahrhundert. Prague / Academia,1973, 88 p.

Tejral 1976 : TEJRAL (J.), Grundzüge der Völkerwanderungszeit in Mähren. Prague / Academia, 1976, $122 \mathrm{p}$

Tejral 1997 : TEJRAL (J.), Neue Aspekte der frühvölkerwanderungszeitlichen Chronologie im Mitteldonauraum, in: TEJRAL (J.), FRIESINGER (H.), KAZANSKI (M.), éd., Neue Beiträge zur Erforschung der Spätantike im mittleren Donauraum. Brno / Archeologický Ústav Akademie Věd České Republiky, 1997, p. 321-392.

Tejral 1997a : TEJRAL (J.), Les fédérés de l'Empire et la formation des royaumes barbares dans la région du Danube moyen à la lumière des données archéologiques. Antiquités Nationales, vol. 29, 1997a, p. 137-166. 
Tejral 2002 : TEJRAL (J.), Beiträge zur Chronologie des langobardischen Fundstoffes nördlich der mittleren Donau, in : TEJRAL (J.), éd., Probleme der frühen Merowingerzeit im Mitteldonauraum. Brno / Archeologický Ústav Akademie Věd České Republiky, 2002, p. 313-358.

Tejral 2008 : TEJRAL (J.), Ein Abriss der frühmerowingerzeitlichen Entwicklung im mittleren Donauraum bis zum Anfang des 6. Jahrhunderts, in: BEMMANNN (J.), SCHMAUDER (M.), éd., Kulturwandel in Mitteleuropa. LangobardenAwaren-Slawen. Bonn / Dr. Rudolf Habelt GhbH, 2008, p. 249-283.
Vallet, Kazanski, De Pirey 1995 : VALLET (F.), KAZANSKI (M.), DE PIREY (D.), Éléments étrangers en Bourgogne dans la deuxième moitié du Ve siècle, in : GAILLARD DE SEMAINVILLE (H.), éd., Les Burgondes, apports de l'archéologie, Dijon / Association pour la connaissance du Patrimoine de Bourgogne, 1995, p. 111-127.

Vogt 1930 : VOGT (E.), Das alamannische Gräberfeld am alten Gotterbarmweg in Basel. Anzeiger für Schweizeirische Altetumskunde. N.F., N 32, 1930, p. 145-164.

Werner 1961 : WERNER (J.), Katalog der Sammlung Diergardt (Völkerwanderungszeitliche Schmuck). Band 1. Berlin: Verlag Gebr. Mann, 1961, 126 p. 